\title{
Caracterisation Partielle d'un Facteur Hypothalamique de Liberation des Hormones Gonadotropes Chez la Carpe (Cyprinus carpio L.) Etude in Vitro
}

\author{
Bernard Breton, Bernard Jalabert, et Claudine Weil \\ avec la collaboration technique de Madame Pierrette Reinaud
}

Institut National de la Recherche Agronomique,

C.N.R.Z., Laboratoire de Physiologie des Poissons, 78350 Jouy en Josas, France

Accepted October 22, 1974

\begin{abstract}
L'influence de différents facteurs sur la secrétion in vitro d'hormone gonadotrope par des hypophyses de Carpe a été étudiée grâce au choix d'un milieu d'incubation favorable. Après filtration sur Séphadex G 25 d'extraits acides réalisés, soit à partir d'hypothalamus, soit à partir de neurohypophyses, une activité de type "releasing factor" a été mise en évidence dans des fractions correspondant à un poids moléculaire inférieur à 5,000. Cette activité se distingue d'une part de celle de l'ocytocine et de la vasopressine et d'autre part de celle de neuroamines: adrénaline, noradrénaline, sérotonine et dopamine, qui ne montrent pas d'activité stimulante propre sur la secrétion gonadotrope in vitro. Par ailleurs, la secrétion de "releasing factor" in vitro par des morceaux d'hypothalamus ne parait pas stimulée de façon significative par l'adjonction de dopamine.

Ces résultats confirment l'existence chez les Poissons d'un facteur hypothalamique actif sur la secrétion gonadotrope, qui pourrait dans l'état actuel des données existantes, être comparable au LH/FSH-RH des Mammifères par sa propriété de stimuler la secrétion de c-GtH par l'hypophyse.
\end{abstract}

The influence of different factors on in vitro secretion of carp pituitary gonadotrophin has been studied in a specific incubation medium. After gel filtration of acid extracts of hypothalamus and neurohypophysis on Sephadex G 25, a "releasing factor" type activity was found in fractions having a molecular weight less than 5000. This activity is different from that of oxytocin, vasopressin and the neuroamines, adrenalin, noradrenalin, serotinine and dopamine which do not stimulate gonadotrophin release from carp pituitary in vitro.

Hypothalamic secretion in vitro of "releasing factor" is not increased when dopamine is added.

These results confirm the existence of a hypothalamic factor which stimulates pituitary gonadotrophin secretion. The possible similarity between this factor and mammalian LH/FSH-RH is discussed.

Chez les Poissons téléostéens comme chez les vertébrés supérieurs, la fonction gonadotrope hypophysaire semble être sous le contrôle de l'hypothalamus. En effet, de nombreux travaux mettent en évidence une relation fonctionnelle entre le système nerveux central et la fonction gonadotrope.

Chez Poecilia reticulata, dans l'hypo- physe déconnectée de l'hypothalamus, les cellules basophiles considérées comme gonadotropes dégénèrent totalement, tandis que les cellules à prolactine restent fonctionnelles (Ball et al., 1965). Il en est de même chez l'Anguille (Olivereau, 1969). Chez le Poisson rouge Carassius auratus (Peter, 1970, 1973) la lésion du noyau latéral du tuber (NLT) entraîne une 
diminution de l'activité de la gamétogenèse. Chez Barbus tor la charge du matériel neurosécrétoire du noyau préoptique (NPO) et du NLT est en phase avec celle des cellules gonadotropes hypophysaires au cours du cycle reproducteur: accumulation importante en période d'ovogenèse et décharge brutale au moment de la fraie (Rai, 1973). Dans le NLT Honma et Tamura (1967) ont pu mettre en évidence des variations de la granulation des fibres neurosecrétrices au cours du cycle reproducteur chez Salvelinus leucomaenis pluvius. Enfin chez Zoarces viviparus Öztan (1966) a fait les mêmes observations; de plus des animaux placés sous éclairement continu en avril, période normale de faible charge en matériel neurosecrétoire, présentent une charge équivalente à celle de la période de fraie en septembre, alors que la situation est inversée en plaçant des animaux à l'obscurité en septembre.

Par contre il n'existe que peu de données concernant la nature des secrétions hypothalamiques agissant sur l'hypophyse; seuls des renseignements histochimiques indiquent que le matériel sécrété par certaines fibres hypothalamiques serait de nature peptidique (Abraham, 1973), mais cependant aucune preuve directe ne permet de lui attribuer un rôle physiologique de type LH/FSHRH comme le fait Rai (1973).

Nous avons démontré la présence dans l'hypothalamus de la Carpe d'un facteur stimulant la secrétion d'hormone gonadotrope par des hypophyses de Carpe in vivo (Breton et Weil, 1973) et in vitro (Breton et al., 1971, 1972).

Nous apportons dans ce travail des précisions sur la nature de ce facteur.

\section{MATERIEL ET METHODES}

Toute l'expérimentation a été réalisée sur des carpes communes (Cyprinus carpio L.), variété miroir, originaire d'un étang de la Brenne, d'un poids moyen de $1 \mathrm{~kg}$, mâles et femelles.

\section{Incubation in Vitro}

Aussitôt après décapitation des animaux, les hypophyses prélevées sont placées dans une solution physiologique isotonique à $4^{\circ}$. Une partie de la neurohypophyse est separée de l'adénohypophyse par dissection sous loupe binocculaire. L'adénohypophyse est alors coupée sagittalement. Puis les demihypophyses sont placées séparement dans des coupelles de verre contenant $5 \mathrm{ml}$ de milieu d'incubation. L'une des demi-hypophyses sert de témoin, l'autre est traitée par les substances à tester.

Les conditions d'incubation permettant d'obtenir des réponses homogènes et répétables ont été recherchées.

Le KRB couramment utilisé pour des incubations de tissus de Mammifères et conçu pour être tamponné sous atmosphère de $5 \%$ de $\mathrm{CO}_{2}$ à $37^{\circ}$, nous a semblé mal adapté à l'incubation de tissus de Poissons (poikilothermes dont la température corporelle et la tension en $\mathrm{CO}_{2}$ dissout du sang sont inférieures). C'est pourquoi nous avons essayé deux autres types de milieux.

MMH, milieu minéral préparé à partir des données de Houston et Madden (1968) (Tableau 1) sur la composition de plasma de Carpes acclimatées pendant plus de 3 semaines à $27^{\circ}$. Ce milieu a été tam-

TABLEAU 1

Concentration des Principaux Cations dans le Plasma des Carpes Utilisées pour nos ExPÉriences (Quelques Jours Seulement APrès Transport et STOCKAGe À $\left.15^{\circ}\right)^{a}$

\begin{tabular}{llcc}
\hline & $\begin{array}{c}\text { Plasma des carpes placées dans nos } \\
\text { conditions expérimentales } \\
\text { (21 échantillons) }\end{array}$ & $\begin{array}{c}\text { Plasma des carpes acclimatées à 27 }{ }^{\circ} \mathrm{C} \\
\text { (d'après Houston et Madden, 1968) } \\
\text { (13 échantillons) }\end{array}$ \\
\hline $\mathrm{Na}$ & $\mathrm{m} M /$ liter & $134 \pm 22$ & $137.3 \pm 2.3$ \\
$\mathrm{~K}$ & $\mathrm{~m} M /$ liter & $8.90 \pm 1.18$ & $2.39 \pm 0.19$ \\
$\mathrm{Mg}$ & $\mathrm{m} M$ /liter & $1.63 \pm 0.07$ & $1.21 \pm 0.05$ \\
$\mathrm{Ca}$ & $\mathrm{m} M /$ liter & $4.16 \pm 0.19$ & $2.44 \pm 0.10$
\end{tabular}

${ }^{a}$ Comparée aux valeurs fournies par Houston et Madden (1968) pour le plasma de Carpes acclimatées à $27^{\circ}$ pendant plus de trois semaines (valeurs moyennes \pm 1 erreur standard). 
ponné par du bicarbonate de sodium sous atmosphère contenant $1 \% \mathrm{CO}_{2}, 49 \% \mathrm{O}_{2}$ et $50 \% \mathrm{~N}_{2}$.

MMC, milieu minéral "Carpe" préparé d'après des résultats d'analyses effectuées sur le plasma de nos propres animaux expérimentaux (Tableau 1). (Le sang est prélevé sur héparinate d'ammonium. Aprés minéralisation du plasma, sa composition ionique a été déterminée par spectrophotométrie de flamme.) Ces valeurs paraissent anormales par rapport à celles de Houston et Madden, probablement à cause du transport récent et de l'état de stress des animaux. La différence fondamentale entre ces milieux réside dans leur teneur en $\mathrm{K}^{+}$, élément dont l'absence peut s'avérer limitante dans les phénomènes de secrétion in vitro. C'est pourquoi nous avons cherché à savoir si de telles différences pouvaient jouer un rôle important dans nos incubations d'hypophyses. Ce milieu a été tamponné selon deux modalités: soit parle bicarbonate de sodium, dans les mêmes conditions que le $\mathrm{MMH}$, sous $1 \% \mathrm{CO}_{2}$ (MMCb), soit par le système Tris-HCL $0.02 M$ sous atmosphère normale (MMCt). La température d'incubation choisie est de $20^{\circ}$.

\section{Substances Testées pour leur}

\section{Activité Stimulante sur la}

Secrétion Gonadotrope

Hypophysaire

Pour apporter des précisions sur la nature du facteur de stimulation hypothalamique, nous avons utilisé 2 types de préparations préparées à partir d'hypothalamus et de neurohypophyses de Carpes par filtration sur gel sephadex G 25 .

Les tissus hypothalamiques et neurohypophysaires sont prélevés dans l'azote liquide, puis transformés en poudre acétonique par broyage et agitation dans l'acétone froid à $4^{\circ}$. Les deux types de poudres obtenues sont soumis à extraction par l'acide acétique $2 N, 24 \mathrm{hr}$ à $4^{\circ}$. Après centrifugation, les deux surnageants sont lyophilisés et sont fractionnés séparement sur la même colonne de séphadex G 25 . L'élution est pratiquée par de l'acide formique $0.1 \mathrm{M}$.

Le diagramme d'élution montre deux pics composites principaux dans les deux cas (Fig. 1). Les fractions correspondant à ces deux pics sont réunies séparement et lyophilisées (fraction A et B). Les 4 échantillons ainsi obtenus ont été utilisés à la dose de $2 \mu \mathrm{g} / \mathrm{ml}$ de milieu.

En utilisant les tests de la variation de pression artérielle (Dekanski, 1952) et de la contraction de la mamelle de ratte in vitro (Smith, 1961), nous avons mis en évidence des activités de type vasopres-

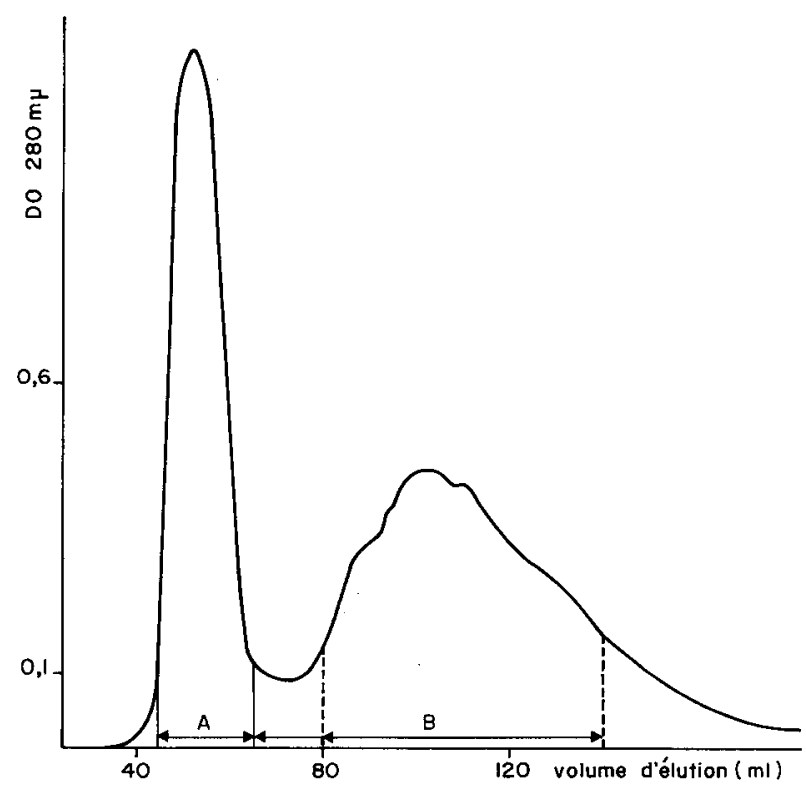

FIG. 1. Diagramme d'élution d'un extrait acide de neurohypophyse de Carpe sur séphadex $\mathrm{G} 25$ en acide formique $0.1 \mathrm{M}$, colonne $1.5 \times 60 \mathrm{~cm}$. 
sique et ocytocique dans la fraction B de l'extrait neurohypophysaire. Ces activités sont de l'ordre de $0.5 \mathrm{IU} / \mathrm{ml}$ d'extrait dans les deux cas. L'action des préparations obtenues a donc été comparée à celle de l'ocytocine et de la vasopressine Sandoz aux doses de 0.1 et $1 \mathrm{IU} / \mathrm{ml}$ de milieu.

L'effet de certaines neuroamines sur la stimulation de la secrétion gonadotrope a en outre été étudié. Les amines suivantes ont été utilisées (il s'agit de produits Koch et Light):

Dopamine seule aux doses de 100$10-5$ et $1 \mu \mathrm{g} / \mathrm{ml}$ de milieu;

Dopamine en présence de fragments d'hypothalamus de Carpe à la dose de $5 \mu \mathrm{g} / \mathrm{ml}$;

Adrénaline aux doses de $100-5$ et 1 $\mu \mathrm{g} / \mathrm{ml}$ de milieu; $\mu \mathrm{g} / \mathrm{ml}$

Noradrénaline aux doses de 5 et 1

Sérotonine aux doses de 5 et $1 \mu \mathrm{g} / \mathrm{ml}$.

La concentration en hormone gonadotrope (c-GtH) de chaque milieu après incubation a été déterminée par dosage radioimmunologique selon la technique déjà décrite (Breton et al., 1971b). La méthode utilisée permet de bonnes déterminations de la c-GtH dans un milieu, dans une gamme comprise entre 0.3 et $100 \mathrm{ng} / \mathrm{ml}$. Les milieux d'incubation à doser ont donc été dilués 5 ou 10 fois afin d'obtenir des valeurs incluses dans cette gamme. La détermination de l'activité des facteurs cités à été réalisée chaque fois sur des lots de 10 demi-hypophyses témoins et expérimentales.

\section{Expression des Résultats}

La secrétion de chaque demi-hypophyse est caractérisée par un coefficient, $\mathrm{S}^{1}$, rapport de la quantité d'hormone secrétée pendant l'incubation finale en présence ou non de produit à tester, à la quantité d'hormone secrétée pendant une incubation précédente de même durée, et en l'absence de tout traitement. La comparaison de ces coefficients entre demi-hypophyses témoins et traitées met en évidence l'activité de la substance analysée.

$\mathrm{S}=1$ correspondrait théoriquement à un taux de secrétion constant d'une période d'incubation à la suivante.

TABLEAU 2

Composition et Caractéristiques des Différents MilieuX D'Incubations Utilisés ${ }^{a}$

\begin{tabular}{|c|c|c|c|c|}
\hline & \multirow{2}{*}{$\begin{array}{c}\text { Krebs-Ringer } \\
\text { bicarbonate } \\
\text { KRB }\end{array}$} & \multirow{2}{*}{$\begin{array}{l}\text { M.M.H. } \\
\text { bicarbonate }\end{array}$} & \multicolumn{2}{|c|}{ M.M.C. } \\
\hline & & & $\begin{array}{c}\text { Tampon bicarbonate } \\
\text { M.M.Cb }\end{array}$ & $\begin{array}{c}\text { Tampon Tris- } \mathrm{HCl} \\
\text { M.M.Ct }\end{array}$ \\
\hline $\mathrm{NaCl}$ (g/liter) & 6.92 & 7.30 & 7.04 & 6.80 \\
\hline $\mathrm{KCl}$ (g/liter) & 0.43 & 0.18 & 0.67 & 0.58 \\
\hline $\mathrm{MgSO}_{4}\left(7 \mathrm{H}_{2} \mathrm{O}\right) \mathrm{g} /$ liter & 0.30 & 0.07 & 0.07 & 0.07 \\
\hline $\mathrm{MgCl}_{2}\left(6 \mathrm{H}_{2} \mathrm{O}\right) \mathrm{g} /$ liter & & 0.18 & 0.26 & 0.22 \\
\hline $\mathrm{CaCl}_{2}\left(2 \mathrm{H}_{2} \mathrm{O}\right) \mathrm{g} /$ liter & 0.37 & 0.35 & 0.61 & 0.54 \\
\hline $\mathrm{NaH}_{2} \mathrm{PO}_{4}$ g/liter & 0.14 & 0.08 & 0.24 & 0.24 \\
\hline $\mathrm{NaH} \mathrm{CO}$ g/liter & 2.1 & 1 & 1 & 1 \\
\hline Glucose g/liter & 2 & 1 & 1 & 1 \\
\hline Tampon Tris- $\mathrm{HCl}$ & - & - & - & $\begin{array}{c}100 \mathrm{ml} \text { tampon } \\
0.2 \mathrm{M} / \mathrm{liter}\end{array}$ \\
\hline Atmophère & $\mathrm{O}_{2} 95 \%-\mathrm{CO}_{2} 5 \%$ & $\begin{array}{l}\mathrm{O}_{2} 49 \% ; \mathrm{CO}_{2} 1 \% \\
\quad \mathrm{~N}_{2} 50 \%\end{array}$ & $\begin{array}{c}\mathrm{O}_{2} 49 \% ; \mathrm{O}_{2} 1 \% \\
\mathrm{~N}_{2} 50 \%\end{array}$ & normale \\
\hline $\mathrm{pH}$ à $25^{\circ}$ & $7.2-7.4$ & $7.4-7.6$ & $7.4-7.6$ & 7.5 \\
\hline p.o. mesurée & 290 & 275 & 285 & 290 \\
\hline
\end{tabular}

${ }^{a}$ Le milieu M.M.H. respecte sensiblement la balance des cations du plasma de Carpe d'après les valeurs de Houston et Madden, et les milieux M.M.C. sont reconstitués d'après nos propres valeurs (Tableau 1). 
Les résultats ont été comparés par analyse de variance.

\section{RESULTATS \\ Influence de la composition du milieu d'incubation sur la sécrétion gonadotrope \\ in vitro}

La composition des différents milieux est donnée dans le tableau 2. Les taux de secrétion gonadotrope des demihypophyses in vitro ont été suivis pendant $6 \mathrm{hr}$, les milieux étant changés toutes les 90 min. Les quantités de c-GtH secrétées, par ml de milieu, ramenées au milligramme d'hypophyse fraîche, sont représentées sur la Fig. 2.

On constate que dans le milieu Kreb Ringer-bicarbonate (KRB) et le milieu physiologique de Carpe tamponné au Tris (MMCt), les niveaux de sécrétion restent hétérogènes apres $6 \mathrm{hr}$ d'incubation et trois changements de milieu. Au contraire, dans le milieu tamponné par du bicarbonate (MMCb) les taux de secrétion sont beaucoup plus homogènes, et à un degré moindre dans le MMH.

Dans le MMCb, les niveaux de sécrétion restent stables après deux renouvellements, c'est-à-dire après $3 \mathrm{hr}$ d'incubation. L'addition d'un extrait hypothalamique dans le milieu d'incubation au 3ème changement de milieu (Fig. 3) se traduit par une élévation homogène des taux de secrétion gonadotrope hypophysaire dans le milieu reconstitué $\mathrm{MMCb}$, alors qu'en tampon Tris- $\mathrm{HCl}$ cette élévation reste hétérogène. Nous avons donc adopté le milieu minéral reconstitué, tamponné au bicarbonate (MMCb).

\section{Influence de la fréquence des changements de milieu}

Nous venons de voir qu'après changement de milieu toutes les $90 \mathrm{~min}$, les niveaux de secrétion se stabilisent après 3

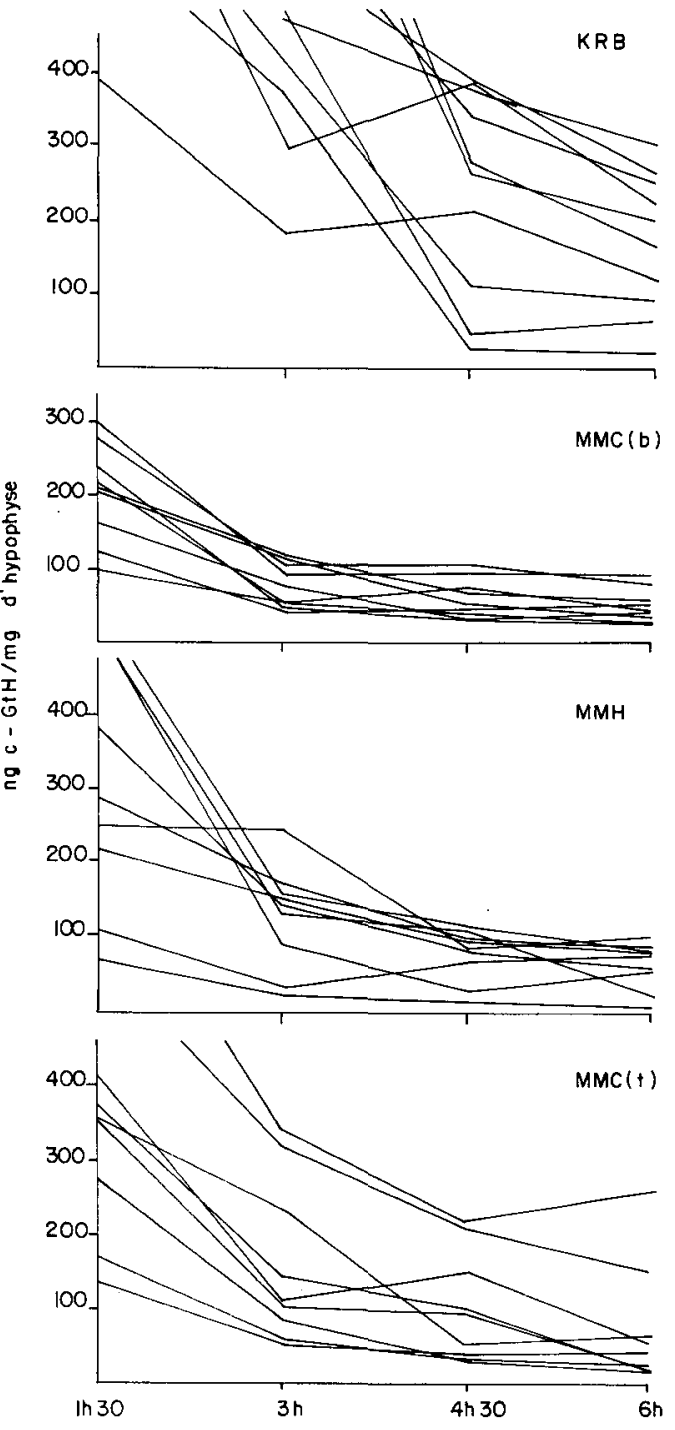

FIG. 2. Effet de différents milieux isotonique d'incubation sur la secrétion de c-GtH in vitro par des demi-hypophyses de Carpe. Chaque courbe représente l'évolution de la secrétion d'une demi-glande.

hr. Sur la Fig. 4 sont portés les résultats obtenus par renouvellement toutes les 30 et $60 \mathrm{~min}$. Quelle que soit la fréquence de changement de milieu, on retrouve la stabilisation des taux de secrétion après $3 \mathrm{hr}$ d'incubation.

Cependant quand la fréquence est de 30 min, on constate de légères fluctuations des niveaux, bien qu'ils soient homogènes dans l'ensemble. 


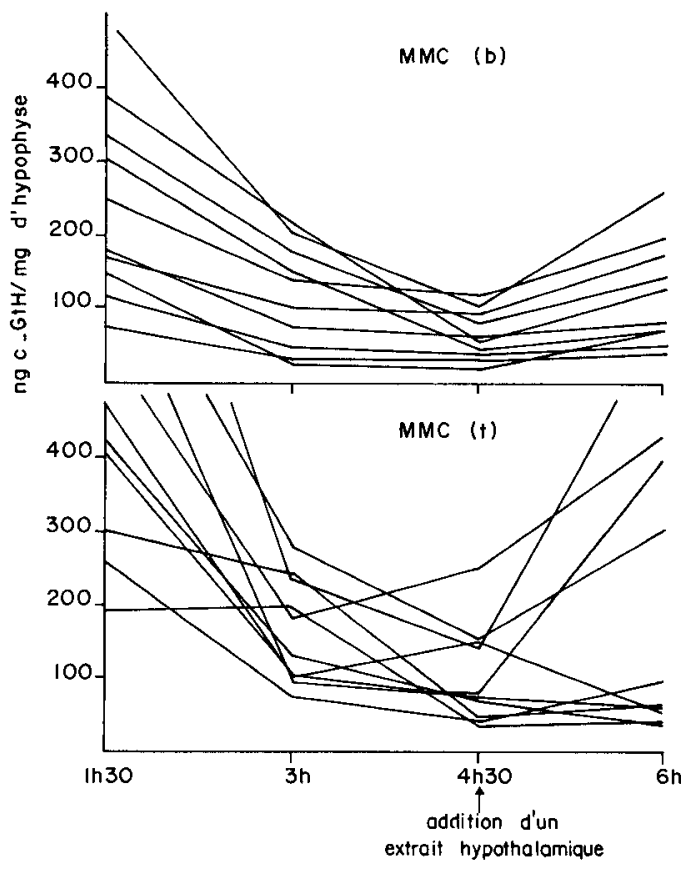

FIG. 3. Effet de l'addition d'extrait hypothalamique sur la secrétion de c-GtH in vitro dans un milieu minéral carpe tamponné au bicarbonate M.M.Cb et au Tris M.M.Ct.
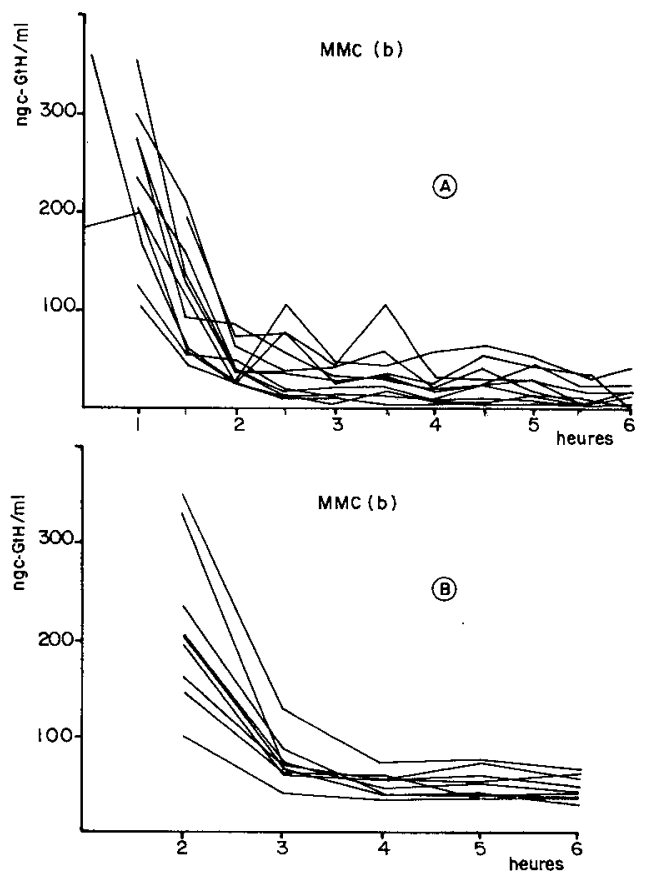

Fig. 4. Effet de la fréquence des changements de milieu sur la secrétion de c-GtH in-vitro: A, changement toutes les $30 \mathrm{~min}$; B, changement toutes les 60 $\min$.
Etude de la sécrétion hypophysaire in vitro sous l'influence de differents facteurs hypophysaires et hypothalamiques

\section{Extraits Hypothalamiques et Neurohypophysaires}

Les fractions A obtenues après filtration sur gel séphadex $G 25$ des extraits acides hypothalamiques et neurohypophysaires n'ont pas provoqué de stimulation de la secrétion gonadotrope hypophysaire in vitro. Par contre, les fractions $\mathrm{B}$ à la dose de $2 \mu \mathrm{g} / \mathrm{ml}$ de milieu entraînent une stimulation. Les coefficients de secrétion passent respectivement de $0.82 \pm 0.25$ à I. $52 \pm 0.53$ et $1.43 \pm 0.36$ avec la préparation hypothalamique, et de $0.77 \pm 0.19$ à $1.94 \pm 0.35$ et $1.67 \pm 0.41$ avec la préparation neurohypophysaire.

Il n'y a pas de différence statistique entre deux répétitions de la même préparation et entre les effets des deux extraits à la même dose.

Les contrôles de l'activité propre de l'ocytocine et de la vasopressine n'ont pas donné de résultats positifs (Fig. 6) aux doses de 0.1 et $1 \mathrm{IU} / \mathrm{ml}$ de milieu.

\section{Action des Neuroamines}

1. Dopamine. Quelle que soit la dose utilisée, la dopamine ne présente pas en elle-même d'activité stimulante sur la secrétion gonadotrope in vitro (Fig. 7). Son action a aussi été testée sur la libération de "releasing factor" à partir d'hypothalamus in vitro. Dans ce but des demi-hypophyses de Carpe ont été incubées en présence d'hypothalamus en morceaux avec addition ou non de dopamine à la dose de $5 \mu \mathrm{g} / \mathrm{ml}$ de milieu.

En l'absence de dopamine deux hypothalamus provoquent une stimulation de la secrétion de c-GtH de $0.75 \pm 0.15$ a $1.34 \pm 0.39$, alors que le coefficient de secrétion est porté à $1.82 \pm 0.38$ (Fig. 8) en présence de dopamine; la différence n'est cependant pas significative au seuil $p=0.05$. 


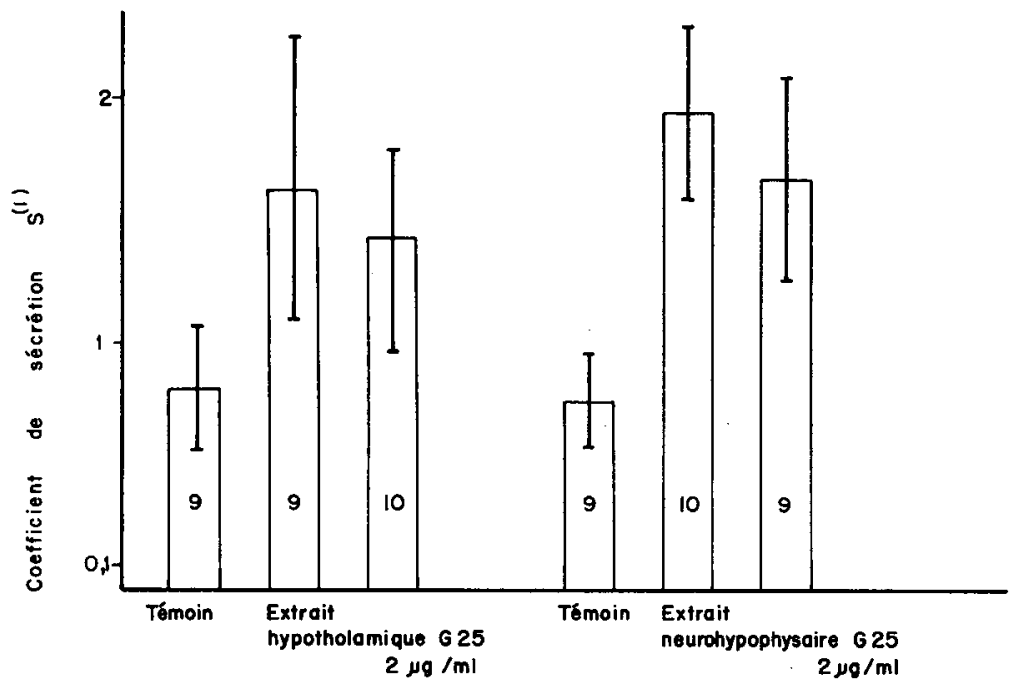

FIG. 5. Comparaison des effets des extraits hypothalamiques et neurohypophysaires après filtration sur séphadex G 25 (intervalle de confiance $p=0.05$ ). Le chiffre à l'intérieur des histogrammes représente le nombre de demi-glandes par essai.

2. Les autres amines utilisées. Adrénaline, noradrénaline, sérotonine n'ont pas montré d'activité stimulante sur la secrétion gonadotrope in vitro. Les effets de ces amines n'ont pas été testés en présence d'hypothalamus.

\section{DISCUSSION}

\section{Milieu d'incubation}

L'utilisation d'un milieu d'incubation spécifique (MMCb) qui parait adapté au tissu hypophysaire de Carpe a permis d'obtenir une homogénéité plus importante des taux de secrétion hypophysaire in vitro, alors que l'utilisation de divers autres milieux parmi lesquels le KRB conduisent à des résultats plus hétérogènes, difficiles à analyser.

Le milieu $\mathrm{MMCb}$ réalisé fortuitement d'après l'analyse de plasma de Carpe en état de stress diffère par ses composantes $\mathrm{K}^{+}$et $\mathrm{Ca}^{2+}$ à la fois du KRB classiquement utilisé pour l'incubation de tissus de Mam-

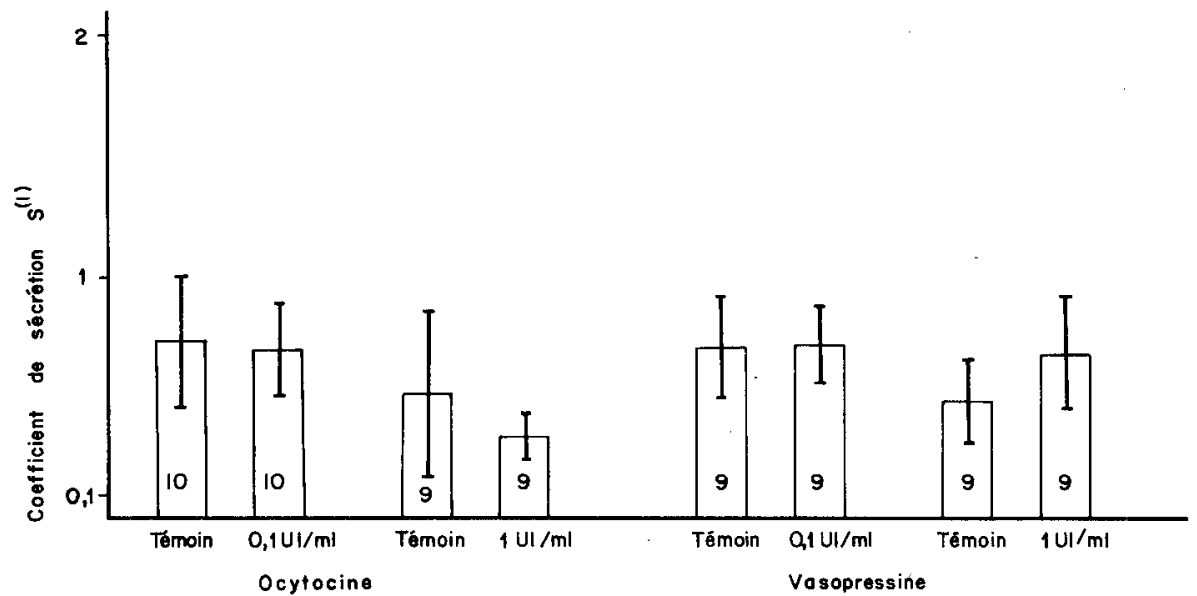

FIG. 6. Effet de l'ocytocine et de la vasopressine sur la secrétion de c-CtH in vitro (intervalle de confiance $p=$ $0.05)$. 


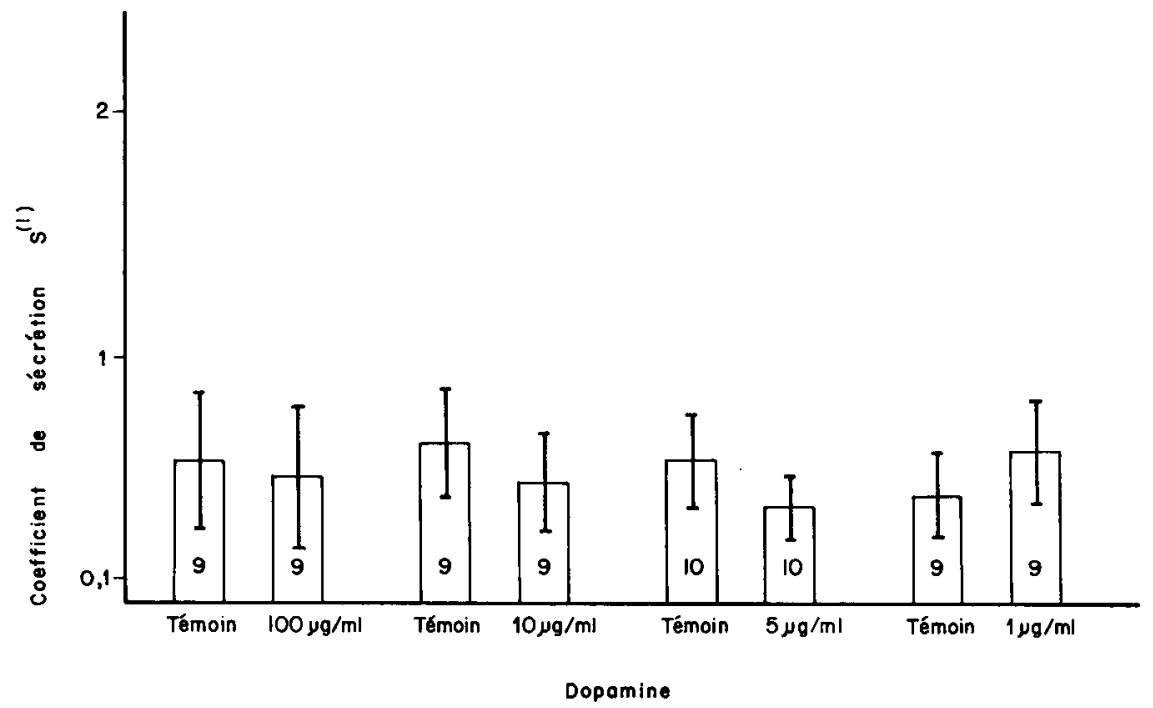

FIG. 7. Effet de la dopamine sur la secrétion de c-GtH in vitro (intergalle de confiance $p=0.05$ ).

mifères et aussi, en fait, des teneurs physiologiques trouvées par Houston et Madden (1968) chez la Carpe. Or, si l'on pouvait attendre qu'un milieu optimum pour un tissu hypophysaire de Poisson soit différent du KRB, il est à priori plus surprenant de constater que l'équilibre ion-

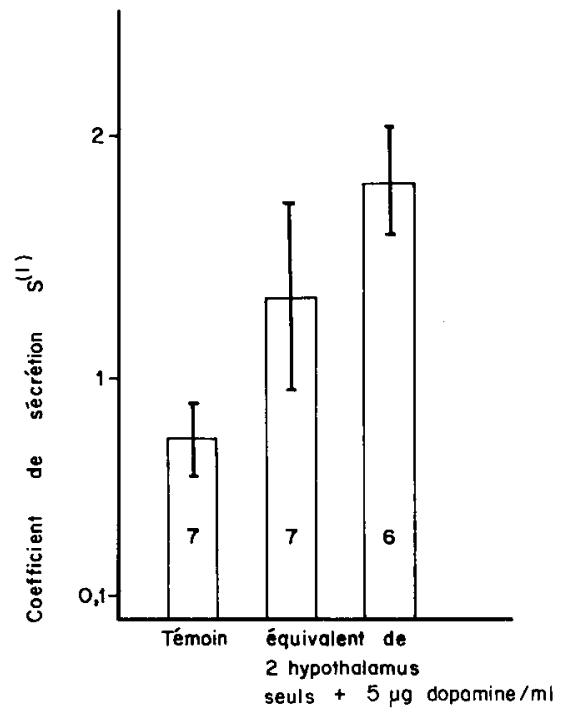

Fig. 8. Stimulation in vitro de la secrétion gonadotrope hypophysaire c-GtH par du tissu hypothalamique en présence ou non de dopamine à la dose de 5 $\mu \mathrm{g} / \mathrm{ml}$ (intervalle de confiance $p=0.05$ ). ique du $\mathrm{MMCb}$ ne corresponde pas aux valeurs plasmatiques physiologiques normales de la Carpe. Il nous semble en fait que cette observation révèle effectivement que les conditions requises pour le fonctionnement normal d'un organe incubé in vitro dans des conditions de survie à court terme ne correspondent pas forcément à l'équilibre plasmatique.

Chez le Rat, la présence de $\mathrm{Ca}^{2+}$ est nécessaire à l'action du FSH-RH in vitro (Wakabayashi et al., 1969); son absence fait disparaître l'activité du FSH-RH sur la synthèse de la FSH. D'autre part, le potassium favorise la secrétion de $\mathrm{LH}$ et $\mathrm{FSH}$, mimant le LH-FSH/RH (Jutisz et de la Llosa, 1970). En présence d'EDTA ou en l'absence de $\mathrm{Ca}^{2+}$, cette action intrinsèque du potassium disparaît (Wakabayashi et McCann, 1970).

L'un de nous (Weil, 1972) a pu démontrer que la stimulation optimale par le $\mathrm{K}^{+}$était obtenue dans du KRB contenant $7.05 \mathrm{mM} /$ litre, c'est-à-dire une concentration en $\mathrm{K}^{+}$proche de la valeur de 8,90 $\mathrm{m} M /$ litre dans le plasma pourrait éventuellement être un facteur limitant. Ainsi l'ajustement des teneurs en $\mathrm{K}^{+}$et $\mathrm{Ca}^{2+} \mathrm{du}$ milieu d'incubation à des valeurs aussi 
TABLEAU 3

Composition en CATIONS des DifFÉRENTS MilieuX UTILISÉs (m $M /$ /iter)

\begin{tabular}{lrrrrr}
\hline & KRB & MMH & MMC(b) & MMC(t) & $\begin{array}{c}\text { Plasma } \\
\text { de Carpe }\end{array}$ \\
\hline $\mathrm{Na}^{+}$ & 143.4 & 137.28 & 134.5 & 118.36 & 134.5 \\
$\mathrm{~K}^{+}$ & 5.87 & 2.40 & 8.9 & 7.78 & 8.9 \\
$\mathrm{Mg}^{2+}$ & 1.24 & 1.20 & 1.6 & 1.38 & 1.6 \\
$\mathrm{Ca}^{2+}$ & 2.52 & 2.40 & 4.15 & 3.67 & 4.16 \\
\hline
\end{tabular}

proches que possible de leurs concentrations dans le plasma des animaux paraît indispensable à l'obtention de taux de secrétion reproductibles d'une hypophyse à l'autre.

Les fluctuations des niveaux de secrétion observées lors des changements de milieu toutes les $30 \mathrm{~min}$ pourraient être dues à des variations du $\mathrm{pH}$ du milieu bicarbonaté qui n'a pas le temps d'atteindre sa valeur d'équilibre dans l'atmosphère d'incubation à $1 \%$ de $\mathrm{CO}_{2}$.

\section{Nature du facteur de stimulation hypothalamique}

Après passage sur séphadex G 25 des extraits hypothalamique et neurohypophysaire, seules les fractions B se sont révélées actives. Ces fractions correspondent à un ensemble de molécules d'un poids moléculaire inférieur à 5,000 . L'allure du pic d'élution (Fig. 1) laisse supposer au moins 4 composantes dans les fractions B parmi lesquelles nous avons pu mettre en évidence les activités pressiques et ocytociques, et l'activité de type "releasing factor." Les deux hormones neurohypophysaires se sont révélées inactives in vitro aux doses utilisées. Il semble donc possible d'écarter l'hypothèse de certains auteurs (Egami et Ishii, 1962) attribuant à ces peptides la fonction de facteurs de stimulation. Cependant les hormones neurohypophysaires mammaliennes différent de celles de Carpe par un résidu d'acide aminé (Acher et al., 1965), et une conclusion définitive sur l'action de ces deux hormones sur la secrétion hypophysaire gonadotrope chez la Carpe ne pourra être tirée qu'après essai des hormones neurohypophysaires spécifiques de l'espèce. Le facteur hypothalamique agissant sur la secrétion gonadotrope hypophysaire in vitro est différent de la dopamine, de l'adrénaline, de la noradrénaline et de la sérotonine. Dans des études sur l'ultrastructure du complexe hypothalamohypophysaire des poissons, de nombreux auteurs ont décrit des contacts neurocellulaires et neurovasculaires d'origine hypothalamiques (Knowles et Vollrath, 1966), autour des cellules de l'antéhypophyse certaines de ces fibres étant de nature aminergique (Zambrano, 1971).

Zambrano (1973) ainsi qu'Abraham (1973) attribuent un rôle prépondérant à ces terminaisons aminergiques dans la décharge des hormones antéhypophysaires; pour Olivereau (1971) les terminaisons sérotoninergiques seraient plus particulièrement impliquées dans le contrôle de la secrétion prolactinique chez l'Anguille.

Nos résultats n'excluent pas une action in vivo indirecte des amines.

Ils ne permettent pas non plus de démontrer l'existence d'un mécanisme dopaminergique contrôlant la secrétion du Gn-RH comme cela a été démontré pour le LH-FSH-RH chez le rat in vitro (Kamberi et McCann, 1969, 1970) et in vivo (Kamberi et al., 1971).

Les deux préparations hypothalamique et neurohypophysaire obtenues après passage sur Séphadex G 25 ont montré des activités de type gonadotropes "releasing factor" équivalentes. Dès 1928, Scharrer avait émis l'hypothèse de l'équivalence physiologique de la neurohypophyse et de l'hypothalamus chez les poissons où les fibres neurosecrétrices originaires des noyaux préoptique et latéral tubérien pénètrent dans la neurohypophyse.

Nos résultats apportent un support quantitatif et qualitatif à cette hypothèse, 
au moins au niveau des secrétions hypothalamiques de type "releasing factor."

En conclusion, le facteur de stimulation gonadotrope hypophysaire d'origine hypothalamique est d'un poids moléculaire inférieur à 5,000, et diffère de l'ocytocine, de la vasopressine et des neuroamines. Il pourrait être proche du LH/FSH-RH mammalien au moins pour son activité biologique, puisque le LH/FSH-RH synthétique provoque in vitro et in vivo (Breton et Weil, 1973) une décharge d'hormone gonadotrope importante chez la Carpe et que l'extrait hypothalamique de poisson entraîne une libération de LH de l'hypophyse de Bélier in vitro (Breton et al., 1972). La détermination de sa nature biochimique reste à faire et suppose que nous disposions d'une quantité de matériel hypothalamique importante et dont la collecte est actuellement en cours.

\section{REMERCIEMENTS}

Nous remercions L. Gueguen de la station de $\mathrm{Nu}$ trition I.N.R.A., Jouy en Josas, pour l'analyse des minéraux du plasma de Carpe et $\mathbf{J}$. Martinet de la station de Physiologie de la Lactation I.N.R.A., Jouy en Josas, pour l'aide qu'il nous a apportée dans la détermination des activités ocytocique et vasopressique des extraits hypothalamiques et neurohypophysaires.

Nous remercions également l'Institut National de la Recherche Agronomique et la D.G.R.S.T. (contrat $\mathrm{n}^{\circ}$ 7I.7.3025) qui ont supporté ce travail.

\section{REFERENCES}

Abraham, M. (1973). The neurosecretory innervation of the pituitary of Mugil cephalus (Teleostei). Abstracts of Paper. 7th Conference of European Comparative Endocrinologists. Budapest, 26-31 août 1973.

Acher, R., Chauvet, J., Chauvet, M. T., and Crepy, D. (1965). Caractérisation des hormones neurohypophysaires d'un poisson osseux d'eau douce, la Carpe (Cyprinus carpio). Comparaison avec les hormones des poissons marins. Comp. Biochem. Physiol. 14, 245-254.

Ball, J. N., Olivereau, M., Slicher, A. M., and Kallman, K. (1965). Functional capacity of ectopic pituitary transplants in the teleost Poecilia formosa with a comparative discussion on the transplant pituitary. Phil. Trans. Ser. B. Biol. Sci. 756, 69-99.
Breton, B., Jalabert, B., Billard, R., and Weil, C. (1971a). Stimulation in vitro de la secrétion d'hormone gonadotrope hypophysaire par un fac. teur hypothalamique chez la Carpe. C. R. Acad. Sci. Sér. D 273, 2591-2594.

Breton, B., Kann, G., Burzawa, G. E., and Billard, R. (1971b). Dosage radioimmunologique d'une hormone gonadotrope de Carpe (Cyprinus carpio L.). C. R. Acad. Sci. Sér. D 272, 1515-1517.

Breton, B., Weil, C., Jalabert, B., and Billard, R. (1972). Activité réciproque des facteurs hypothalamiques de Bélier et de Poissons téléostéens sur la secrétion in vitro des hormones gonadotropes c-GtH et o-LH respectivement par des hypophyses de Carpe et de Bélier. C. R. Acad. Sci. Ser. $D$ 274, 2530-2533.

Breton, B., and Weil, C. (1973). Effets du LH/FSHRH synthétique et d'extraits hypothalamiques de Carpe sur la secrétion d'hormone gonadotrope in vivo chez la Carpe (Cyprinus carpio L.). C. $R$. Acad. Sci. Sér. D 277, 2061-2064.

Dekanski, J. (1952). The quantitative assay of vasopressin. Brit. J. Pharmacol. 7, 567-572.

Egami, E., and Ishii, S. (1962). Hypophysial control of reproductive functions in teleost fishes. Gen. Comp. Endocrinol. Suppl. 1, 248-253.

Honma, Y., and Tamura, E. (1967). Studies on Japanese chars of the genus Salvelinus. IV. The hypothalamo-hypophysial vascular system of the Nikko iwana; Salvelinus leuconaenis pluvius in relation to its neurosecretory system. Bull. Jap. Soc. Sci. Fisheries 33, 303-310.

Houston, H. A., and Madden, J. A. (1968). Environmental temperature and plasma electrolyte regulation in the Carp Cyprinus carpio. Nature (London) 217, 969-970.

Jutisz, M., and de la Llosa, P. (1970). Requirement of $\mathrm{Ca}^{++}$and $\mathrm{Mg}^{++}$ions for the in vitro release of follicle stimulating hormone from rat pituitary glands and its subsequent biosynthesis. Endocrinology 86, 761-768.

Kamberi, I. A., and McCann, S. M. (1969). Effets of biogenic amines, FSH releasing factor (FRF) and other substances on the release of FSH by pituitaries incubated in vitro. Endocrinology 85, 815-824.

Kamberi, I. A., Mical, R. S., and Porter, J. C. (1969). Luteinizing hormone releasing activity in hypophysial stalk blood and elevation by dopamine. Science 166, 388-390.

Kamberi, I. A., Scheiner, H. P. G., and McCann, S. M. (1970). Action of Dopamine to induce release of RSH releasing factor (FRF) from hypothalamic tissues in vitro. Endocrinology 86, 278-284.

Kamberi, I. A., Mical, R. S., and Porter, J. C. (1971). Effects of anterior pituitary perfusion and in- 
traventricular injection of catecholamine on FSH release. Endocrinology 88, 1003-1011.

Knowles, F., and Vollrath, L. (1966). Neurosecretory innervation of the pituitary of the eel Anguilla and Conger. II. The structure and innervation of the pars distalis at different stages of the life cycle. Phil. Trans. Roy. Soc. London Ser. B. 250, 329-342.

Olivereau, M. (1969). Cytologie de l'hypophyse autotransplantée chez l'Anguille; comparaison avec celle de Poecilia. J. Neuroendocrinol. 927, 252-260.

Olivereau, M. (1971). Action de la réserpine chez l'Anguille. I. Cellulee à prolactine de l'hypophyse du mâle. Z. Zellforsch. Mikrosk. Anat. 121, 232-243.

Öztan, N. (1966). The structure of the hypothalamic neurosecretory cells of Zoarces viviparus $\mathbf{L}$. under the conditions of constant dark and light during the reproductive cycle. Z. Zellforsch. $\mathbf{7 5}$, 66-82.

Peter, R. E. (1970). Hypothalamic control of thyroid gland activity and gonadal activity in the goldfish Carassius auratus. Gen. Comp. Endocrinol. 14, 334-355.

Peter, R. E. (1973). Neuroendocrinology of teleosts. Amer. Zool. 13, 743-755.

Rai, B. P. (1973). On the neurosecretory centres in the hypothalamus and their relationship in reproduction in Tor (Barbus) tor. Acta Anat. 85, 429-433.

Scharrer, R. (1928). Die Lichtempfindlichkeit blinder ebritzen (Untersuchungen über das Zwisch en hum der Fische I). Z. Physiol. 7, 1-38.

Smith, M. W. (1961). Some properties of rat mammary tissue. Nature (London) 190, 541-542.

Wakabayashi, K., Kamberi, I. A., and McCann, S. M. (1969). In vitro response of the rat pituitary to gonadotrophin releasing factor and ions. Endocrinology 85, 1046-1056.

Wakabayashi, K., and McCann, S. M. (1970). In vitro responses of anterior pituitary glands for normal, castrated and androgen. Treated male rats to $\mathrm{LH}$ releasing factor (LRF) and high potassium medium. Endocrinology 87, 771-778.

Weil, C. (1972). Rapport D. E. A. Université de Paris VI.

Zambrano, D. (1971). The nucleus lateralis tuberis system of the Gobiid fish Gillichthys mirabilis. III. Functional modification of the neurons and gonadotropic cells. Gen. Comp. Endocrinol. 17, 164-182.

Zambrano, D., and Iturriza, F. C. (1973). Hypothalamic hypophysial relationships in the south American Lungfish Lepidosiren paradoxa. Gen. Comp. Endocrinol. 20, 256-275. 